\title{
Tanshinone IIA inhibits human gastric carcinoma AGS cell growth by decreasing BiP, TCTP, Mcl-1 and Bcl-xL and increasing Bax and CHOP protein expression
}

\author{
CHIN-CHENG SU
}

\begin{abstract}
Tumor Research Center of Integrative Medicine; Comprehensive Breast Cancer Center; Department of Surgery, Changhua Christian Hospital, Changhua 50006; School of Chinese Medicine, College of Chinese Medicine, China Medical University, Taichung 40402, Taiwan, R.O.C.
\end{abstract}

Received April 1, 2014; Accepted September 22, 2014

DOI: 10.3892/ijmm.2014.1949

\begin{abstract}
Tanshinone IIA (Tan-IIA) is extracted from Danshen (Salviae Miltiorrhizae Radix) and is a natural anti-cancer agent, which possesses antitumor activity in a variety of human cancer cells. Tan-IIA can induce apoptosis and inhibit the proliferation of gastric cancer through different molecular mechanisms. However, the efficacy and molecular mechanism of Tan-IIA in gastric cancer have not been well studied. In the present study, the cytotoxicity of Tan-IIA in human gastric cancer AGS cells by 3-(4,5-dimethylthiazol-2-y1)-2,5-diphenyltetrazolium bromide assay was examined. The protein expression levels of B-cell lymphoma-extra large (Bcl-xL), Bcl-2-associated $\mathrm{X}$ protein (Bax), myeloid cell leukemia 1 protein (Mcl-1), translationally-controlled tumor protein (TCTP), binding immunoglobulin protein (BiP), calnexin, protein kinase-like endoplasmic reticulum kinase, eIF2 $\alpha$, activating transcription factor 4 (ATF4), inositol-requiring enzyme $1 \alpha$ (IRE1 $\alpha$ ), ATF6, caspase-12, caspase-9, caspase-3, C/EBP-homologous protein $(\mathrm{CHOP})$ and $\beta$-actin in AGS cells were measured by western blot analysis. The results showed that Tan-IIA inhibited AGS cells in a time-and dose-dependent manner. AGS cells treated with Tan-IIA upregulated the protein expression of caspase-12, caspase- 9 , caspase-3, CHOP and Bax, but downregulated the protein expression of BiP, TCTP, Mcl-1 and Bcl-xL. These findings indicated that Tan-IIA inhibits the growth of human gastric cancer AGS cells. One of the molecular mechanisms may be through decreasing the protein
\end{abstract}

Correspondence to: Dr Chin-Cheng Su, Tumor Research Center of Integrative Medicine, Comprehensive Breast Cancer Center, Changhua Christian Hospital, 135 Nan-Hsiao Street, Changhua 50006, Taiwan, R.O.C.

E-mail: succ.maeva@msa.hinet.net

Key words: tanshinone IIA, gastric carcinoma AGS cells, endoplasmic reticulum stress, translationally-controlled tumor protein, myeloid cell leukemia 1 protein expression of $\mathrm{BiP}$ to induce the activation of endoplasmic reticulum stress, followed by increasing the protein expression of caspase-12 to upregulate CHOP expression. The other may be through decreasing the protein expression of Mcl-1, Bcl-xL and TCTP, but increasing Bax, caspase- 9 and caspase- 3 to induce apoptosis. The chemotherapeutic potential of Tan-IIA for human gastric cancer warrants further study in the future.

\section{Introduction}

Tanshinone IIA (Tan-IIA; $\mathrm{C}_{19} \mathrm{H}_{18} \mathrm{O}_{3}$ ), a phenanthrenequinone derivative extracted from Danshen, Salviae Miltiorrhizae Radix $(1,2)$, is a natural anti-cancer agent, which possesses antitumor activity in a variety of human cancer cells, such as lung (3), colon (4) and breast cancer (5). It has been well documented that Tan-IIA can induce apoptosis and reverse the malignant phenotype of SGC7901 gastric cancer cells. Tan-IIA also exerted powerful inhibitory effects in the gastric cancer cells, SGC7901, in a time- and dose-dependent manner, and arrested gastric cancer cells in the $\mathrm{G}_{0} / \mathrm{G}_{1}$ phase $(6,7)$. Tan-IIA induced growth inhibition and apoptosis in gastric cancer in vitro and in vivo. Tan-IIA not only arrested gastric cancer MKN-45 cells in $\mathrm{G}_{2} / \mathrm{M}$ phase, but also triggered the intrinsic apoptotic-signaling pathway, through upregulating expression of the 553 gene and downregulating the expression of the B-cell lymphoma (Bcl) 2 gene $(8,9)$. These studies indicate that Tan-IIA may serve as an effective adjunctive reagent in the treatment of gastric cancer. However, the molecular mechanisms of Tan-IIA in gastric cancer cells remain unclear. In previous studies by my group, it was shown that Tan-IIA inhibited the growth of pancreatic cancer BxPC-3 cells by decreasing the protein expression of translationally-controlled tumor protein (TCTP), myeloid cell leukemia 1 protein (Mcl-1) and Bcl-extra large (Bcl-xL) (10). Tan-IIA inhibited the growth of breast cancer cells, BT-20, through increasing the protein expression of caspase-12, GADD153 and phospho-p38 (11). Tan-IIA also inhibited the growth of hepatocellular carcinoma Hep-J5 cells by increasing calreticulin, caspase-12 and GADD153 protein expression (12). However, the molecular mechanisms that cause Tan-IIA to induce apoptosis in human gastric cancer via interaction of endoplasmic reticulum 
stress (ER stress) and intrinsic apoptotic signaling pathway have not been clarified. In the present study, the effects of Tan-IIA in human gastric cancer AGS cells were investigated.

\section{Materials and methods}

Materials. The AGS human gastric adenocarcinoma cell line (BCRC no.: 60102) was obtained from the Food Industry Research and Development Institute (Hsinchu, Taiwan). Tan-IIA (CAS-no.: 568-72-9), 3-(4,5-dimethylthiazol-2-y1)-2,5-diphenyltetrazolium bromide (MTT), sodium deoxycholate, leupeptin, Triton X-100, Tris-HCl, ribonuclease-A, sodium pyruvate, 4-(2-hydroxethyl)-1-piperazineethanesulphonic acid, dimethylsulfoxide (DMSO) and Tween-20, mouse anti- $\beta$-actin were obtained from Sigma-Aldrich (St. Louis, MO, USA). Potassium phosphate and $0.2 \mathrm{~mm}$ polyvinylidene fluoride membranes were purchased from Merck KGaA (Darmstadt, Germany). F-12K medium, fetal bovine serum (FBS), penicillin-streptomycin and glutamine were obtained from Gibco-BRL (Carlsbad, CA, USA). BioMax film was obtained from Kodak. The Bcl-2-associated X protein (Bax) [no.: 2774; molecular weight (MW) $20 \mathrm{kDa}$ ], Bcl-xL (no.: 2764; MW $30 \mathrm{kDa}$ ), Mcl-1 (no.: 5453; MW 40 kDa), TCTP (no.: 8441; MW 23 kDa), binding immunoglobulin protein (BiP) (no.: 3177; MW $78 \mathrm{kDa}$ ), calnexin (no.: 2679; MW $90 \mathrm{kDa}$ ), protein kinase-like endoplasmic reticulum kinase (PERK) (no.: 5683; MW $140 \mathrm{kDa}$ ), eIF2 $\alpha$ (no.: 9722; MW $38 \mathrm{kDa}$ ), inositol-requiring enzyme $1 \alpha$ (IRE1 $\alpha$ ) (no.: 3294; MW $130 \mathrm{kDa}$ ), caspase-12 (no.: 2202; MW $42 \mathrm{kDa}$ ), caspase-9 (no.: 9502; MW $35 \mathrm{kDa}$ ), caspase-3 (no.: 9661; MW $17 \mathrm{kDa}$ ), ERK (no.: 4370; MW 44/42 kDa), and p38 (no.: 4511; MW $40 \mathrm{kDa}$ ) antibodies were all obtained from Cell Signaling Technology, Inc. (Beverly, MA, USA). C/EBP-homologous protein (CHOP) (NB600-1335; MW 29 kDa), p53 (NB100-92601; MW 43 kDa) and c-Jun N-terminal kinase (NB100-192, MW $42 \mathrm{kDa}$ ) antibodies were obtained from Novus Biologicals (Littleton, CO, USA). Activating transcription factor 4 (ATF4) (ab1371; MW 38 kDa) and ATF6 (ab11909; MW 75 kDa) antibodies were obtained from Abcam (Cambridge, MA, USA).

Cell culture. The human gastric adenocarcinoma AGS cells were obtained from the Food Industry Research and Development Institute. The AGS cells were placed into $75-\mathrm{cm}^{2}$ tissue culture flasks and maintained in F-12K with $10 \%$ heat-inactivated FBS, $100 \mathrm{U} / \mathrm{ml}$ penicillin and $100 \mu \mathrm{g} / \mathrm{ml}$ streptomycin. Cells were grown at $37^{\circ} \mathrm{C}$ in a humidified atmosphere of $5 \% \mathrm{CO}_{2}$. All the data presented are from at least three independent experiments.

Cytotoxicity assay. The cytotoxicity of Tan-IIA for AGS cells was evaluated by the MTT assay in triplicate as previously described (13). Briefly, the AGS cells were plated in 96-well plates at a density of $2 \times 10^{4}$ cells/well for $16-20 \mathrm{~h}$. After this the cells were treated with various concentrations $(0,1,3,9,15,30$ and $60 \mu \mathrm{g} / \mathrm{ml}$ ) of Tan-IIA for 24, 48 and $72 \mathrm{~h}$. Subsequently, the cells were incubated with $1 \mathrm{mg} / \mathrm{ml}$ of MTT in fresh complete F-12K medium for $1 \mathrm{~h}$. The surviving cells converted MTT to formazan by forming a blue-purple color when dissolved in DMSO. The intensity of formazan was measured at $590 \mathrm{~nm}$ using a microplate reader. The relative percentage of cell viability was calculated by dividing the absorbance of treated cells by that of the control in each experiment, using the following formula: Proliferation rate $(\%)=(\mathrm{OD}$ test $-\mathrm{OD}$ blank) x 100, where OD test and OD blank are the optical density of the test substances and the blank control, respectively.

Western blot analysis. The western blot analysis procedures are as previously described $(14,15)$. Briefly, AGS cells were treated with various concentrations of Tan-IIA for different durations, and the cells were lysed in the ice-cold whole cell extract buffer containing the protease inhibitors. The lysate was agitated for $30 \mathrm{~min}$ at $4^{\circ} \mathrm{C}$ and centrifuged at $12,281 \mathrm{x} \mathrm{g}$ for $10 \mathrm{~min}$. Protein concentration was measured by the bicinchoninic acid protein assay kit (Pierce Biotechnology, Inc., Rockford, IL, USA). Equal amounts of proteins were subjected to electrophoresis using $12 \%$ sodium dodecyl sulfate-polyacrylamide gels. To verify equal protein loading and transfer, proteins were transferred to polyvinylidene difluoride membranes and the membranes were blocked for $1 \mathrm{~h}$ at $4^{\circ} \mathrm{C}$ using blocking buffer (5\% skimmed dried milk in solution containing $50 \mathrm{mM}$ Tris- $\mathrm{HCl}(\mathrm{pH} 8.0), 2 \mathrm{mM} \mathrm{CaCl}_{2}$, $80 \mathrm{mM}$ sodium chloride, $0.05 \%$ Tween- 20 and $0.02 \%$ sodium azide). The membranes were subsequently incubated for $2 \mathrm{~h}$ at room temperature with the specific primary antibody followed by anti-rabbit or anti-mouse immunoglobulin G-horseradish peroxidase-conjugated secondary antibodies. The membranes were washed three times for $10 \mathrm{~min}$ with washing solution. Finally, the protein bands were visualized on the X-ray film using the enhanced chemiluminescence detection system (PerkinElmer Life and Analytical Sciences, Inc., Boston, MA, USA).

Statistical analysis. Values are presented as the means \pm standard deviation. The Student's t-test was used to analyze statistical significance. $\mathrm{P}<0.05$ was considered to indicate a statistically significant difference for all the tests.

\section{Results}

Effects of Tan-IIA in the viability of AGS cells. The results revealed that Tan-IIA inhibited the proliferation of AGS cells in a time- and dose-dependent manner. The half maximal inhibitory concentration $\left(\mathrm{IC}_{50}\right.$ ) was $5.5,3.7$ and $3.5 \mu \mathrm{g} / \mathrm{ml}$ at 24, 48 and $72 \mathrm{~h}$, respectively (Fig. 1).

Effects of various concentrations of Tan-IIA on the protein expression of Bax, Bcl-xL, Mcl-1, TCTP and $\beta$-actin in AGS cells. The AGS cells were treated with various concentrations of Tan-IIA $(0,2.0,3.7$ and $5.5 \mu \mathrm{g} / \mathrm{ml})$ for 24 or $48 \mathrm{~h}$ and the protein expression levels of Bax, Bcl-xL, Mcl-1, TCTP and $\beta$-actin were evaluated by western blot analysis. The results revealed that Tan-IIA increased the protein expression levels of Bax (Fig. 2A), but significantly decreased Bcl-xL (Fig. 2B), Mcl-1 (Fig. 2C) and TCTP (Fig. 2D) levels.

Effects of various concentrations of Tan-IIA on the protein expression of BiP, calnexin, PERK, eIF2 $\alpha, A T F 4, I R E 1 \alpha$, ATF6, caspase-12, caspase-9, caspase-3, CHOP and $\beta$-actin in AGS cells. The AGS cells were treated with various 


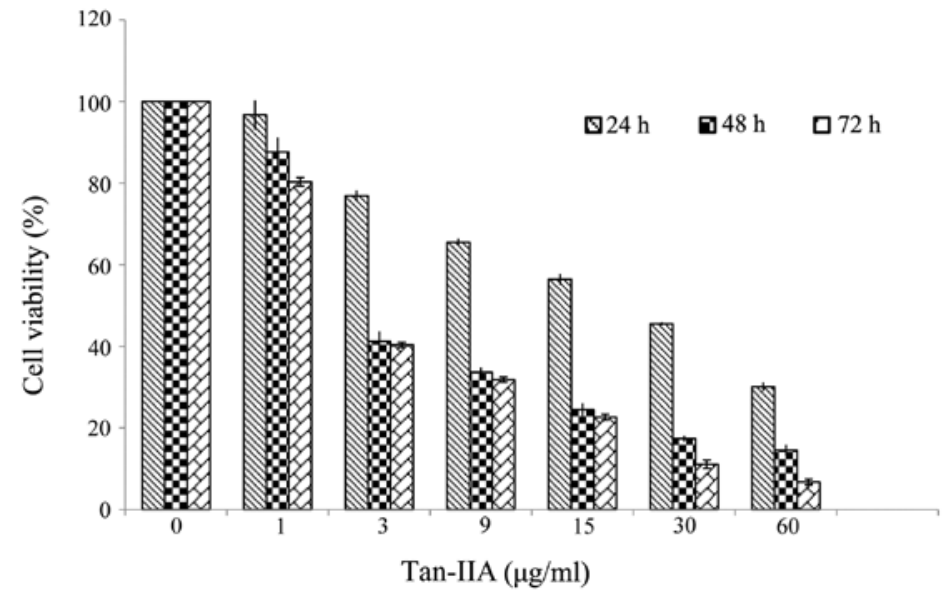

Figure 1. Cytotoxic effects of tanshinone IIA (Tan-IIA) on AGS cells. The cytotoxic effects of Tan-IIA on AGS cells were determined by the 3-(4,5-dimethylthiazol-2-y1)-2,5-diphenyltetrazolium bromide (MTT) assay as described in 'Materials and methods'. Each point is the mean \pm standard deviation of three experiments.

A
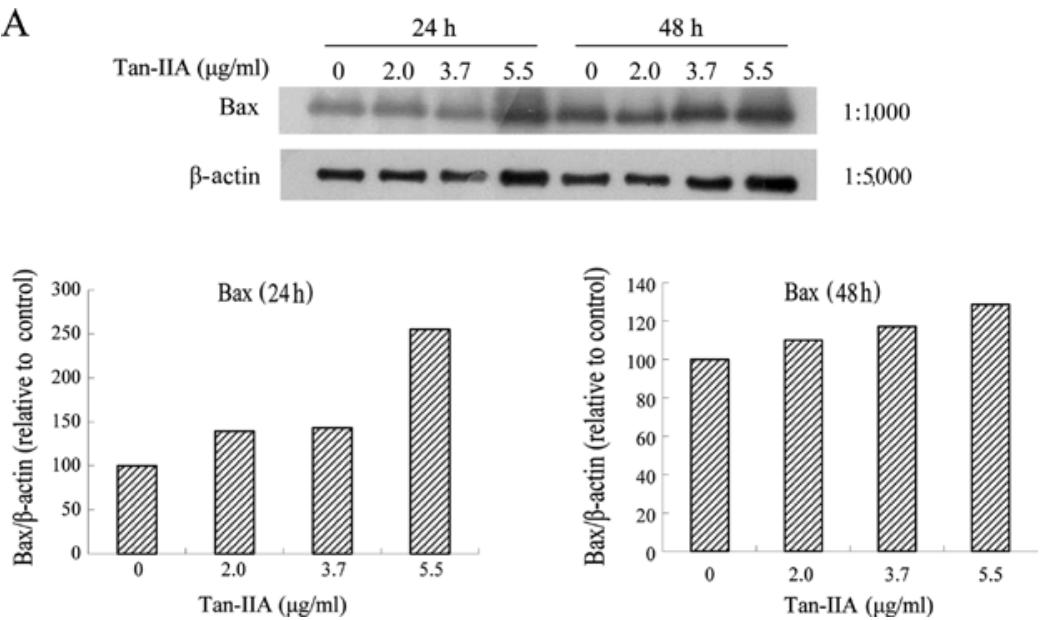

B
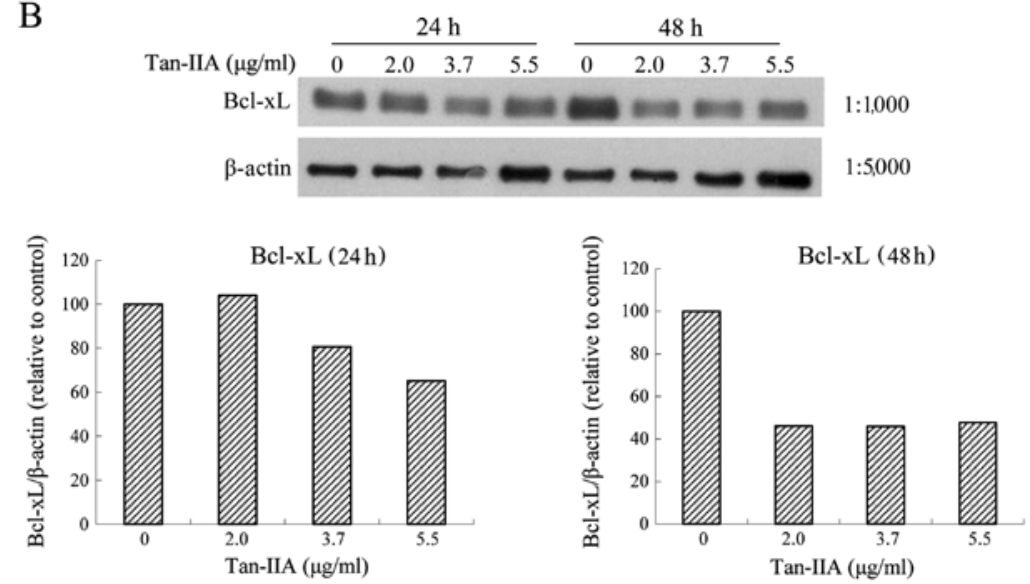

Figure 2. Protein expressions of B-cell lymphoma (Bcl)-2 associated X protein (Bax), Bcl-extra large (xL), myeloid cell leukemia 1 protein (Mcl-1), translationally-controlled tumor protein (TCTP) and $\beta$-actin in AGS cells. The AGS cells were treated with various concentrations of tanshinone IIA (Tan-IIA) (0, 2.0, 3.7 and $5.5 \mu \mathrm{g} / \mathrm{ml}$ ) for 24 or $48 \mathrm{~h}$ and the protein expression levels were evaluated by western blot analysis as described in 'Materials and methods'. The results revealed that Tan-IIA increased the protein expression levels of (A) Bax but significantly decreased those of (B) Bcl-xL in a dose-dependent manner.

concentrations of Tan-IIA $(0,2.0,3.7$ and $5.5 \mu \mathrm{g} / \mathrm{ml})$ for 24 or $48 \mathrm{~h}$ and the protein expression levels of BiP, calnexin, PERK, eIF2 $\alpha$, ATF4, IRE1 $\alpha$, ATF6, caspase-12, caspase-9, caspase- 3 , CHOP and $\beta$-actin were evaluated by western blot analysis. The results revealed that Tan-IIA can decrease the protein expression level of BiP (Fig. 3A) but increased caspase-12 (Fig. 4A), caspase-9 (Fig. 4B), caspase-3 (Fig. 4C), and CHOP (Fig. 4D) levels significantly. The protein expression levels of calnexin, PERK, eIF2 $\alpha$, ATF4, IRE1 $\alpha$ and ATF6 did not change significantly (data not shown). 

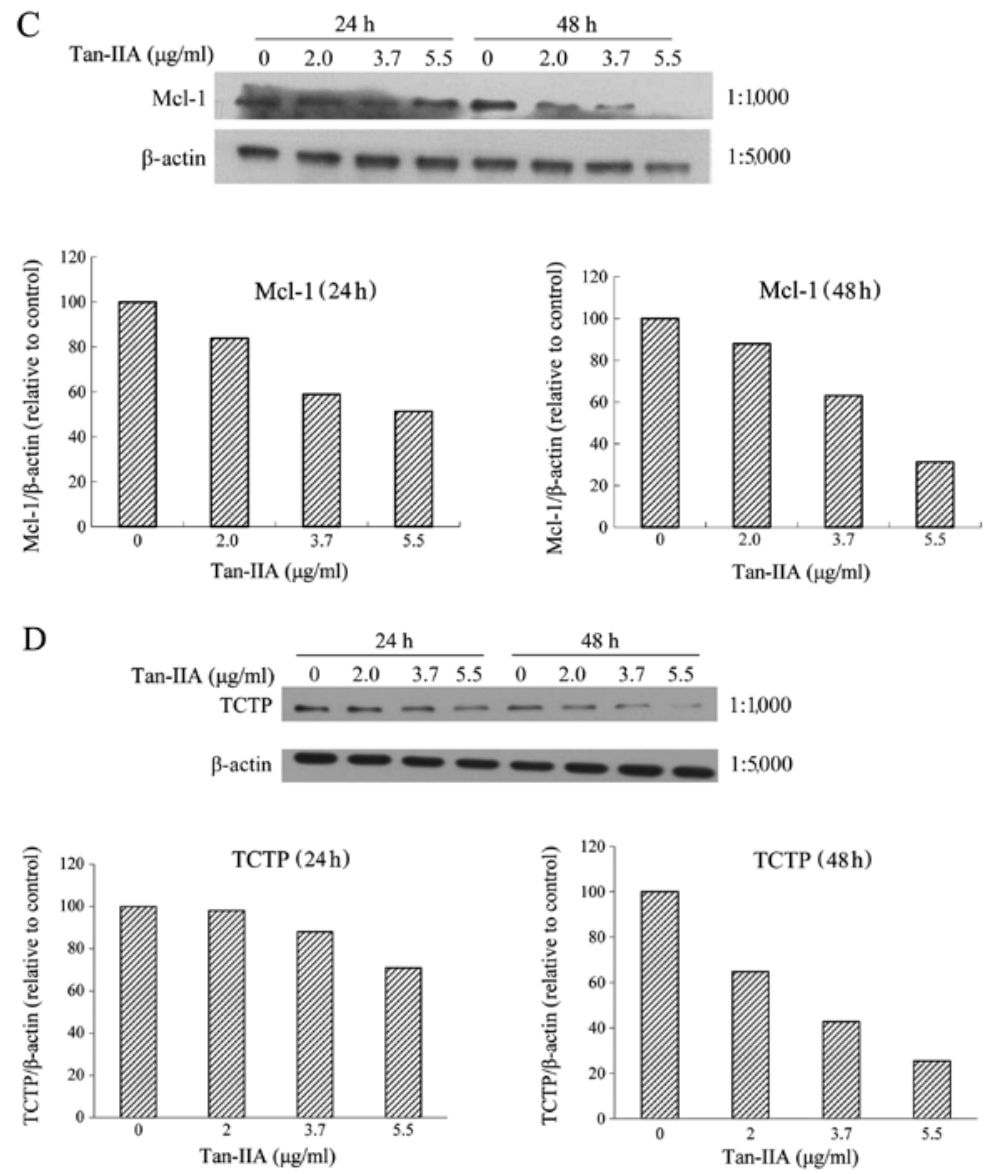

Figure 2. Continued. Protein expression levels of (C) myeloid cell leukemia 1 protein (Mcl-1) and (D) translationally-controlled tumor protein (TCTP) were significantly decreased in a dose-dependent manner.

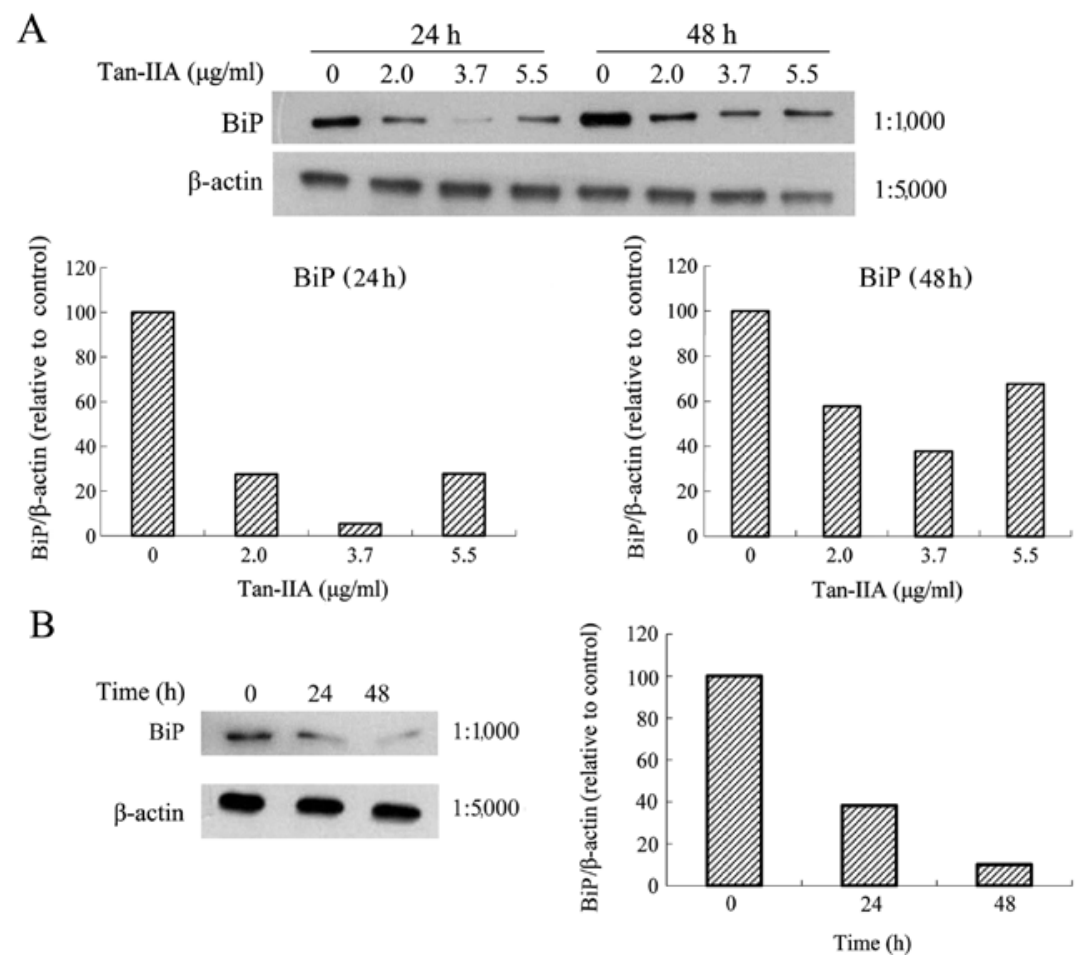

Figure 3. Protein expressions of binding immunoglobulin protein (BiP) and $\beta$-actin in AGS cells. (A) The AGS cells were treated with various concentrations of tanshinone IIA (Tan-IIA) $(0,2.0,3.7$ and $5.5 \mu \mathrm{g} / \mathrm{ml})$ for 24 or $48 \mathrm{~h}$ and the protein expression levels were evaluated by western blot analysis as described in 'Materials and methods'. The results revealed that Tan-IIA can decrease the protein expression level of BiP in a dose-dependent manner. (B) The AGS cells were treated with Tan-IIA $(3.7 \mu \mathrm{g} / \mathrm{ml})$ for different durations $(0,24$ and $48 \mathrm{~h})$ and the protein expression levels were evaluated by western blot analysis as described in 'Materials and methods'. The results revealed that Tan-IIA decreased the protein expression level of BiP in a time-dependent manner. 
A
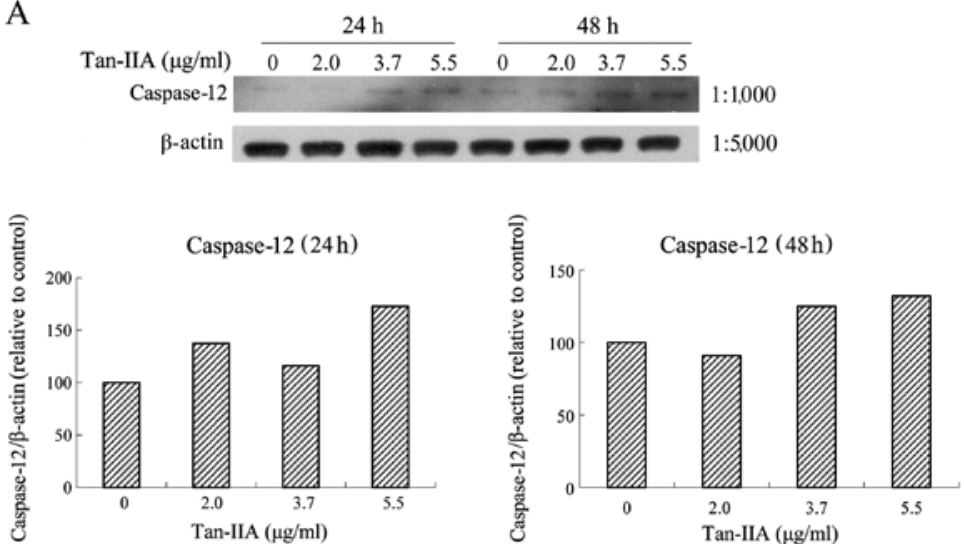

B
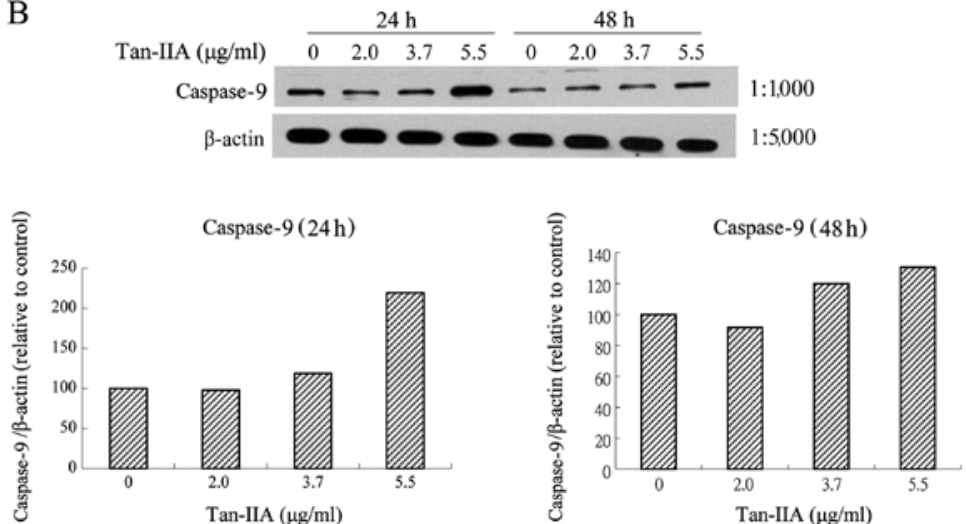

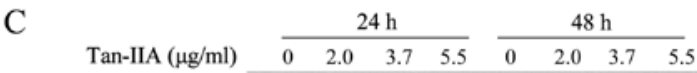

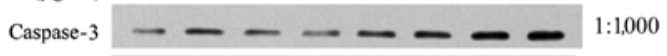

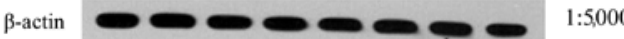
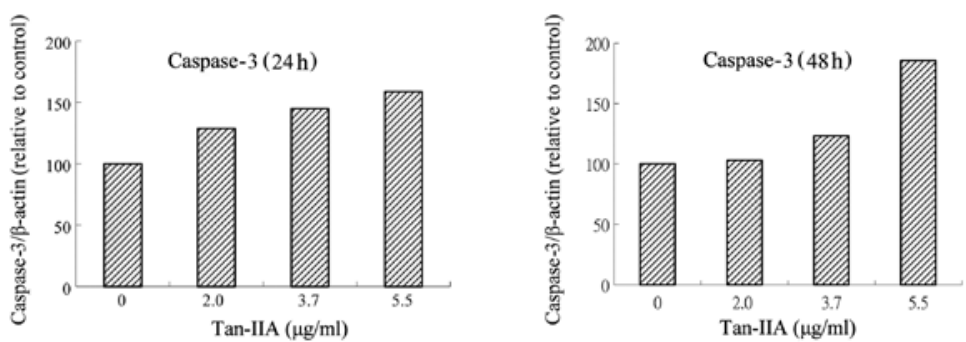

D

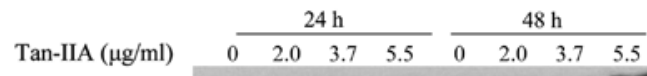

СHOP $-=-m=-\infty: 1,000$

$\beta$-actin

$1: 5,000$
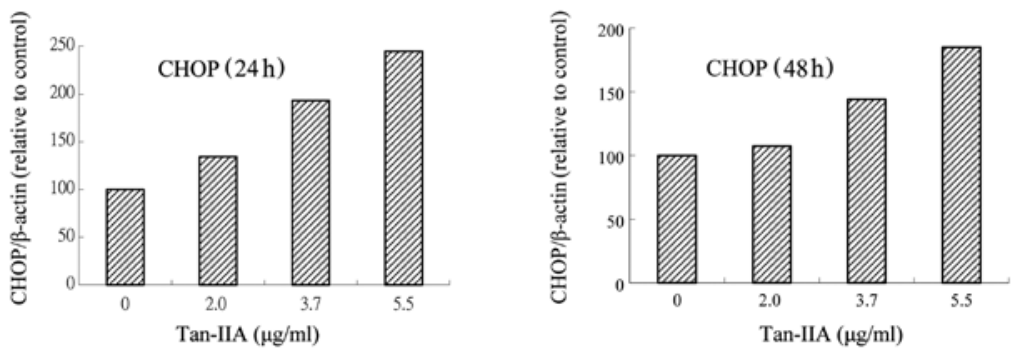

Figure 4. Protein expressions of binding immunoglobulin protein (BiP), calnexin, protein kinase-like endoplasmic reticulum kinase (PERK), eIF2 $\alpha$, activating transcription factor 4 (ATF4), inositol-requiring enzyme $1 \alpha$ (IRE1 $\alpha$ ), ATF6, caspase-12, caspase-9, caspase-3, C/EBP-homologous protein (CHOP) and $\beta$-actin in AGS cells. The AGS cells were treated with various concentrations of tanshinone IIA (Tan-IIA) $(0,2.0,3.7$ and $5.5 \mu \mathrm{g} / \mathrm{ml})$ for 24 or $48 \mathrm{~h}$ and the protein expression levels were evaluated by western blot analysis as described in 'Materials and methods'. The results revealed that Tan-IIA significantly increased (A) caspase-12, (B) caspase-9, (C) caspase-3 and (D) CHOP levels in a dose-dependent manner. 
A
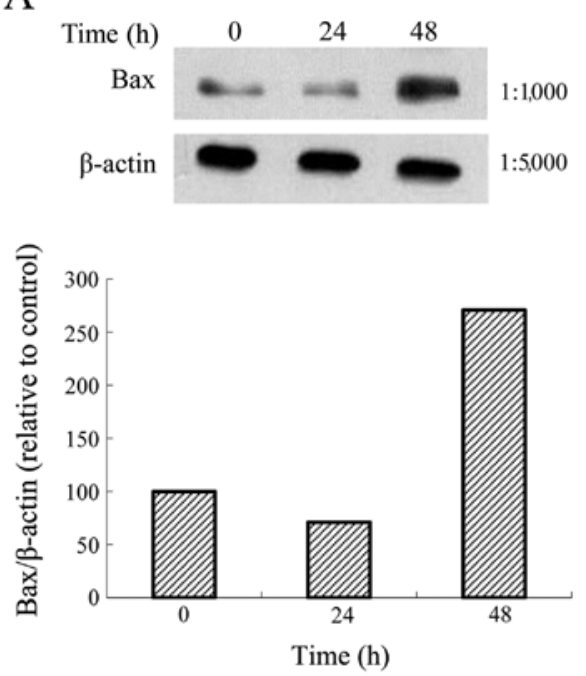

$\mathrm{C}$
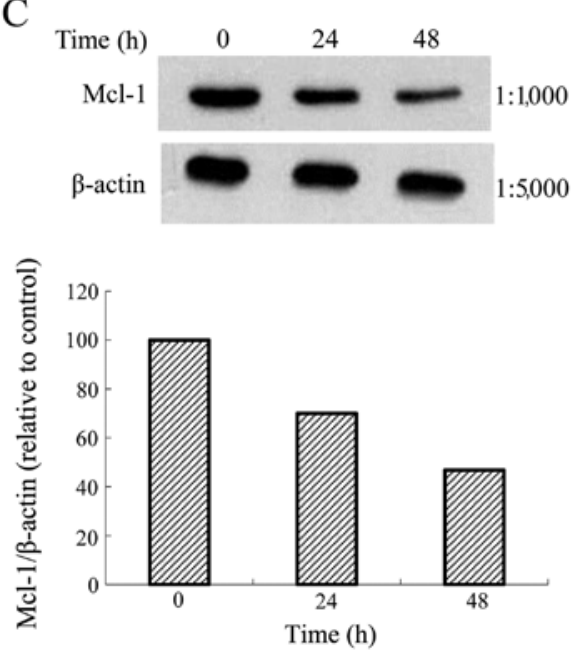

$\mathrm{B}$
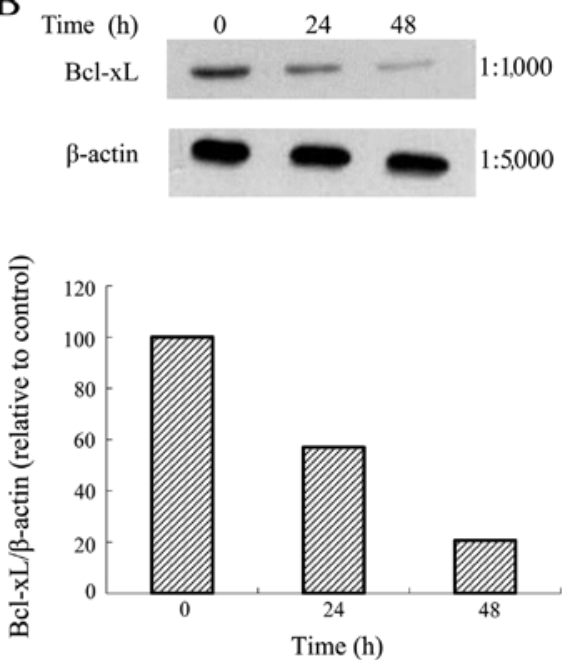

$\mathrm{D}$
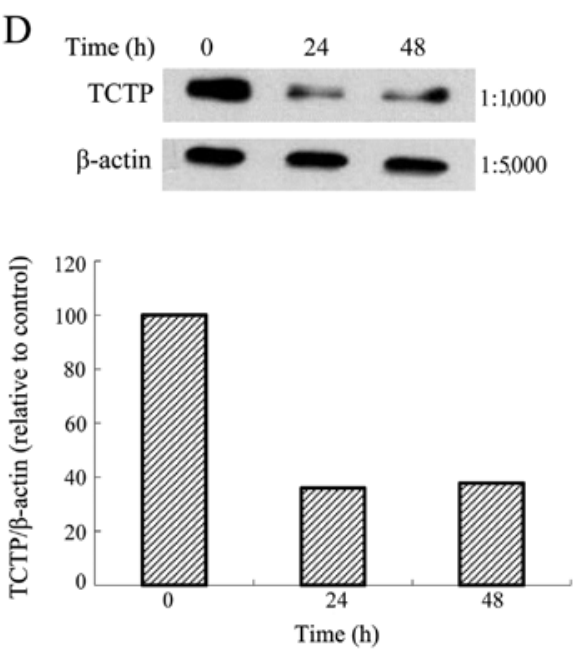

Figure 5. Protein expressions of B-cell lymphoma (Bcl)-2 associated X protein (Bax), myeloid cell leukemia 1 protein (Mcl-1), translationally-controlled tumor protein (TCTP), Bcl-extra large (xL) and $\beta$-actin in AGS cells. The AGS cells were treated with tanshinone IIA (Tan-IIA) $(3.7 \mu \mathrm{g} / \mathrm{ml})$ for different durations ( 0 , 24 and $48 \mathrm{~h}$ ) and the protein expression levels were evaluated by western blot analysis as described in 'Materials and methods'. The results revealed that Tan-IIA increased the protein expression levels of (A) Bax but significantly decreased (B) Bcl-xL, (C) Mcl-1 and (D) TCTP levels in a time-dependent manner.

Effects of one Tan-IIA concentration on the protein expression of Bax, Bcl-xL, Mcl-1, TCTP and $\beta$-actin in AGS cells. The AGS cells were treated with Tan-IIA $(3.7 \mu \mathrm{g} / \mathrm{ml})$ for different durations $(0,24$ and $48 \mathrm{~h})$ and subsequently the protein expression levels of Bax, Bcl-xL, Mcl-1, TCTP and $\beta$-actin were evaluated by western blot analysis. The results revealed that Tan-IIA increased the protein expression levels of Bax (Fig. 5A) but decreased Bcl-xL (Fig. 5B), Mcl-1 (Fig. 5C) and TCTP (Fig. 5D) levels significantly.

Effects of one Tan-IIA concentration on the protein expression of BiP, calnexin, PERK, eIF2 $\alpha$, ATF4, IRE1 $\alpha$, ATF6, caspase-12, caspase-9, caspase-3, CHOP and $\beta$-actin in AGS cells. The AGS cells were treated with Tan-IIA $(3.7 \mu \mathrm{g} / \mathrm{ml})$ for different durations $(0,24$ and $48 \mathrm{~h})$ and the protein expression levels of BiP, calnexin, PERK, eIF2 $\alpha$, ATF4, IRE1 $\alpha$, ATF6, caspase-12, caspase-9, caspase- 3 , CHOP and $\beta$-actin were evaluated by western blot analysis. The results revealed that Tan-IIA can decrease the protein expression level of BiP (Fig. 3B) but increased caspase-12 (Fig. 6A), caspase-9 (Fig. 6B), caspase-3 (Fig. 6C), and CHOP (Fig. 6D) levels significantly.

\section{Discussion}

The results of the present study revealed that Tan-IIA inhibited the proliferation of human gastric cancer AGS cells in a time- and dose-dependent manner. This is in accordance with previous studies (6-9). TCTP was discovered in Ehrlich ascites tumor cells (16), is conserved in all eukaryotes and encodes for a hydrophilic protein of $18-23 \mathrm{kDa}$ (17). TCTP has been implicated in the protection of cells against apoptosis (18). Susini et al (19) showed that TCTP protects from apoptotic cell death by antagonizing Bax function. Liu et al (20) also documented that TCTP stabilized and enhanced the antiapoptotic activity of Mcl-1. These results indicate that TCTP binds to Mcl-1, antagonizing Bax, and thus inhibiting the induction of apoptosis. The present results demonstrated that the treatment of AGS cells with Tan-IIA decreased the protein expression levels of Mcl-1, Bcl-xL and TCTP, but increased Bax, caspase-9 and caspase-3 levels with a time- and dose-dependent manner. Therefore, one of the molecular mechanisms of Tan-IIA involved in the inhibition of human gastric cancer AGS cell proliferation may be through decreasing the protein expression 

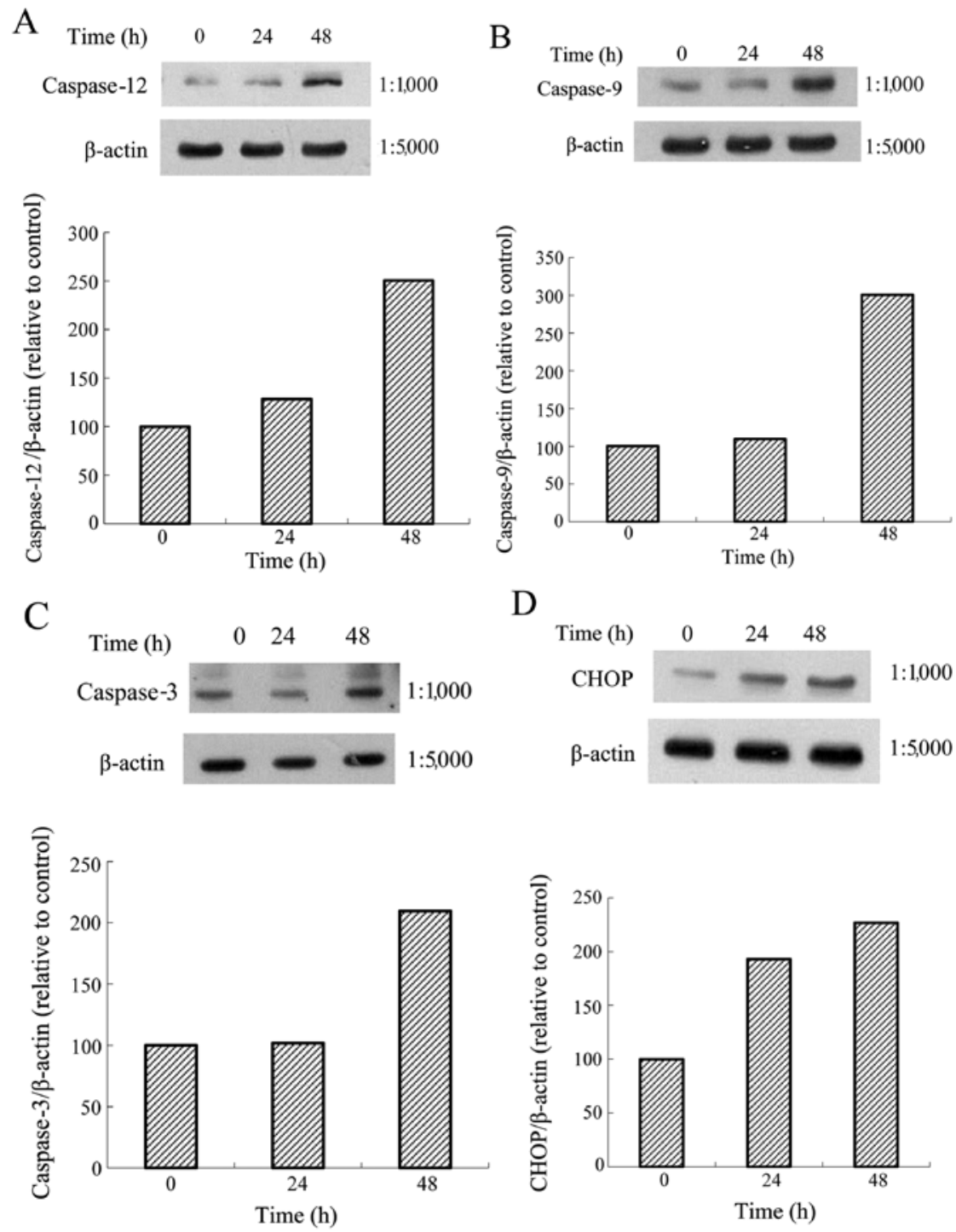

Figure 6. Protein expressions of calnexin, protein kinase-like endoplasmic reticulum kinase (PERK), eIF2 $\alpha$, activating transcription factor 4 (ATF4), inositol-requiring enzyme $1 \alpha$ (IRE1 $\alpha$ ), ATF6, caspase-12, caspase-9, caspase-3, C/EBP-homologous protein (CHOP) and $\beta$-actin in AGS cells. The AGS cells were treated with tanshinone IIA (Tan-IIA) $(3.7 \mu \mathrm{g} / \mathrm{ml})$ for different durations $(0,24$ and $48 \mathrm{~h})$ and the protein expression levels were evaluated by western blot analysis as described in 'Materials and methods'. The results revealed that Tan-IIA significantly increased (A) caspase-12, (B) caspase-9, (C) caspase-3 and (D) CHOP levels in a time-dependent manner.

of Mcl-1, Bcl-xL and TCTP, but increasing Bax, caspase-9 and caspase-3, thus inducing apoptosis. Following endoplasmic reticulum response over loading, ER stress is activated, and the upstream element, caspase-12, is activated to increase the target protein, CHOP (also known as GADD153) (21). Our previous study showed that Tan-IIA induced ER stress to inhibit human breast cancer BT-20 cells (11) and human hepatocellular cancer Hep-J5 cells (12). In the present study, the results showed that Tan-IIA increased the protein expression levels of caspase-12, caspase-9, caspase- 3 and CHOP. These results indicate that Tan-IIA induced ER stress to inhibit the proliferation of AGS cells. The proposed model of the interactions between Bax, TCTP, Mcl-1, Bcl-xL and the ER stress pathway in AGS cells treated with Tan-IIA is shown in Fig. 7.

To the best of our knowledge, this is the first study to demonstrate that Tan-IIA inhibited human gastric cancer AGS cells. One of the molecular mechanisms may be through decreasing the protein expression of $\mathrm{BiP}$ to induce the activation

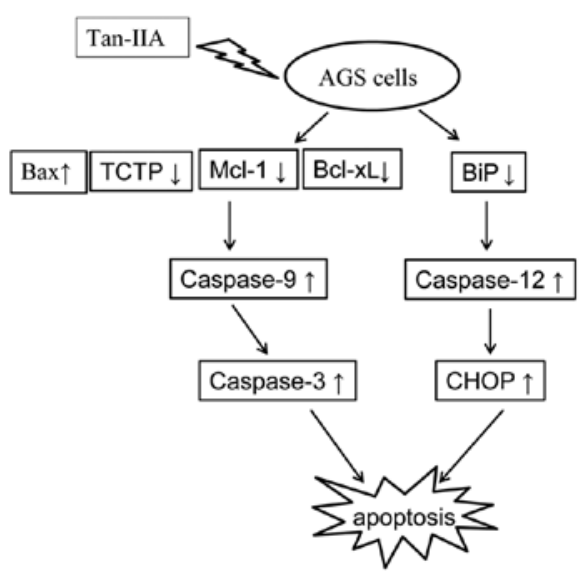

Figure 7. Proposed signaling pathway through which tanshinone IIA (Tan-IIA) exerts its anti-proliferative effects on human gastric cancer AGS cells. Bax, B-cell lymphoma (Bcl)-2 associated X protein; TCTP, translationally-controlled tumor protein; Mcl-1, myeloid cell leukemia 1; Bcl-xL, Bcl-extra large; $\mathrm{BiP}$, binding immunoglobulin protein; CHOP, C/EBP-homologous protein. 
of ER stress, followed by increasing the protein expression of caspase-12 to upregulate $\mathrm{CHOP}$ expression. The other may be through decreasing the protein expression of Mcl-1, Bcl-xL and TCTP, but increasing Bax, caspase- 9 and caspase-3.

\section{Acknowledgements}

The present study was supported by grant no. 102-CCH-IRP-066 from the Research Section of the Changhua Christian Hospital, Changhua, Taiwan, R.O.C.

\section{References}

1. Che AJ, Zhang JY, Li CH, Chen XF, Hu ZD and Chen XG: Separation and determination of active components in Radix Salviae miltiorrhizae and its medicinal preparations by nonaqueous capillary electrophoresis. J Sep Sci 27: 569-575, 2004.

2. Zhou L, Zuo Z and Chow MS: Danshen: an overview of its chemistry, pharmacology, pharmacokinetics, and clinical use. J Clin Pharmacol 45: 1345-1359, 2005.

3. Chiu TL and Su CC: Tanshinone IIA induces apoptosis in human lung cancer A549 cells through the induction of reactive oxygen species and decreasing the mitochondrial membrane potential. Int J Mol Med 25: 231-236, 2010.

4. Su CC, Chen GW, Kang JC and Chan MH: Growth inhibition and apoptosis induction by tanshinone IIA in human colon adenocarcinoma cells. Planta Med 74: 1357-1362, 2008.

5. Su CC and Lin YH: Tanshinone IIA inhibits human breast cancer cells through increased Bax to Bcl-xL ratios. Int J Mol Med 22: 357-361, 2008.

6. Hou J, He J, Jin X, Hu T and Zhang Y: Study on optimisation of extraction process of tanshinone IIA and its mechanism of induction of gastric cancer SGC7901 cell apoptosis. Afr J Tradit Complement Altern Med 10: 456-458, 2013.

7. Xu M, Cao FL, Li NY, Liu YQ, Li YP and Lv CL: Tanshinone IIA reverses the malignant phenotype of SGC7901 gastric cancer cells. Asian Pac J Cancer Prev 14: 173-177, 2013.

8. Chen J, Shi DY, Liu SL and Zhong L: Tanshinone IIA induces growth inhibition and apoptosis in gastric cancer in vitro and in vivo. Oncol Rep 27: 523-528, 2012.
9. Dong X, Dong J and Peng G: Growth-inhibiting and apoptosis-inducing effects of Tanshinone II A on human gastric carcinoma cells. J Huazhong Univ Sci Technolog Med Sci 27: 706-709, 2007.

10. Huang CY, Chiu TL, Kuo SJ, Chien SY, Chen DR and Su CC: Tanshinone IIA inhibits the growth of pancreatic cancer BxPC-3 cells by decreasing protein expression of TCTP, MCL-1 and Bcl-xL. Mol Med Rep 7: 1045-1049, 2013.

11. Yan MY, Chien SY, Kuo SJ, Chen DR and Su CC: Tanshinone IIA inhibits BT-20 human breast cancer cell proliferation through increasing caspase 12, GADD153 and phospho-p38 protein expression. Int J Mol Med 29: 855-863, 2012.

12. Cheng CY and Su CC: Tanshinone IIA inhibits Hep-J5 cells by increasing calreticulin, caspase 12 and GADD153 protein expression. Int J Mol Med 26: 379-385, 2010.

13. Mossman T: Rapid colorimetric assay for cellular growth and survival: application to proliferation and cytotoxicity assays. J Immunol Methods 65: 55-63, 1983.

14. Bradford MM: A rapid and sensitive method for the quantitation of microgram quantities of protein utilizing the principle of protein-dye binding. Anal Biochem 72: 248-254, 1976.

15. Chen HC, Hsieh WT, Chang WC and Chung JG: Aloe-emodin induced in vitro G2/M arrest of cell cycle in human promyelocytic leukemia HL-60 cells. Food Chem Toxicol 42: 1251-1257, 2004.

16. Yenofsky R, Cereghini S, Krowczynska A and Brawerman G: Regulation of mRNA utilization in mouse erythroleukemia cells induced to differentiate by exposure to dimethyl sulfoxide. Mol Cell Biol 3: 1197-1203, 1983.

17. Bommer UA, Lazaris-Karatzas A, De Benedetti A, et al: Translational regulation of the mammalian growth-related protein P23: involvement of eIF-4E. Cell Mol Biol Res 40: 633-641, 1994.

18. Bommer UA and Thiele BJ: The translationally controlled tumour protein (TCTP). Int J Biochem Cell Biol 36: 379-385, 2004.

19. Susini L, Besse S, Duflaut D, et al: TCTP protects from apoptotic cell death by antagonizing bax function. Cell Death Differ 15: 1211-1220, 2008 .

20. Liu H, Peng HW, Cheng YS, Yuan HS and Yang-Yen HF: Stabilization and enhancement of the antiapoptotic activity of mcl-1 by TCTP. Mol Cell Biol 25: 3117-3126, 2005.

21. Ma Y and Hendershot LM: The role of the unfolded protein response in tumour development: friend or foe? Nat Rev Cancer 4: 966-977, 2004. 